\title{
A New Approach to Nonlinear Tracking Control Based on Fuzzy Approximation
}

\author{
Z. Du, T.-C. Lin, V.E. Balas
}

\author{
Zhenbin Du \\ Yantai University, \\ Yantai, Shandong 264005, China \\ E-mail: zhenbindu@yahoo.com.cn \\ Tsung-Chih Lin \\ Feng-Chia University \\ Taichung, 40724, Taiwan \\ E-mail: tclin@fcu.edu.tw \\ Valentina E. Balas \\ Aurel Vlaicu University \\ B-dul Revolutiei 77 \\ 310130 Arad, Romania \\ E-mail: balas@drbalas.ro
}

\begin{abstract}
The problem of tracking control is addressed for a class of nonlinear systems with uncertainties. The original nonlinear systems are approximated by a fuzzy T-S model based on which a state-feedback controller is constructed by using the linear matrix inequalities. The approximating error is eliminated by an adaptive compensator based on fuzzy logic systems. The effectiveness of the proposed control scheme is demonstrated by a simulation example. The main advantage is that the designer makes milder constraint assumption for the approximation error and the uncertainties in nonlinear systems.

Keywords: fuzzy T-S model; fuzzy logic systems; nonlinear systems; uncertainties; tracking control.
\end{abstract}

\section{Introduction}

The problem of controller design for the nonlinear systems with uncertainties is a challenging work. One of effective tools used to solve the problem is fuzzy method. There are two frequently used fuzzy models: fuzzy T-S model [1-13] and fuzzy logic systems [16-17]. Fuzzy T-S model is usually used to approximate nonlinear systems, and it has been widely applied to analyze the stability of nonlinear systems [1-4]. The contributions of these works are very important, but these works could be further improved by a simpler and more practical control scheme. The approximating error is neglected in [1-4], which impacts the stability of the system. Therefore, the designed controller can't always guarantee the stability of the original system. To overcome the effect of the approximating error, some relaxed stability methods are developed in [5-8]. These methods improve the approximation accuracy for nonlinear system. However, the original nonlinear system is still neglected in [5-8]. In order to further relax the effect of the approximating error, it is assumed to satisfy the matching condition in [9-11] and have a upper bound in [1213]. However, the matching condition and the upper bound are not easy to be found in practice, which adds some difficulties to the controller design. The uncertainties in nonlinear systems are assumed to satisfy the constraint of the upper bound in [14-15]. However, the upper bound may be too large or doesn't exist. On the other hand, fuzzy logic systems have been proved to have 
the universal approximation property. By constructing a set of fuzzy "IF-THEN" rules, fuzzy logic system is used to model uncertain nonlinear systems [16-17].

There exist some conservatism of pure fuzzy T-S model in dealing with the approximating error. The pure adaptive fuzzy control also has some shortages of excessively depending on the chosen membership functions. Therefore, it is more interesting to combine both fuzzy models to overcome their shortages each other.

Based on the above discussions, fuzzy T-S model and fuzzy logic systems are combined to design a new tracking-control scheme for a class of nonlinear systems with uncertainties in this paper. A fuzzy T-S model is used to approximate the nonlinear system based on which a statefeedback controller is constructed by use of the linear matrix inequalities. The approximating error and the uncertain nonlinear parts are eliminated by a compensator based on fuzzy logic systems. The main advantages are summarized as follows:

Firstly, fuzzy T-S model and fuzzy logic systems are combined to develop a controller. Compared with the existing works based on fuzzy T-S model [1-13], the proposed method in this paper makes milder constraint assumptions for the approximating error. Secondly, the dimension of the matrix inequalities is reduced, thus, the difficulty of solving the matrix equalities is relaxed. Thirdly, the existing works based on fuzzy logic systems [16-17] excessively depend on the chosen membership functions which are improved in the proposed method of this paper. Finally, the developed controller makes full use of the advantages of two fuzzy models. As a result, it is more convenient to implement the controller in practice.

The rest of the paper is organized as follows. Section 2 provides preliminaries and the formulation of the problem. Section3 develops a procedure of the controller design. Section 4 presents a simulation example of 2-link manipulator to illustrate the effectiveness of the proposed method. These are followed by conclusions in Section 5 .

\section{Problem formulation}

Consider the following nonlinear systems with uncertainties

$$
\begin{aligned}
& \dot{x}_{1}=x_{2}, \\
& \ldots \\
& \dot{x}_{\left(\beta_{1}-1\right)}=x_{\beta_{1}}, \\
& \dot{x}_{\beta_{1}}=f_{1}(x, u)+\tilde{f}_{1}(x, u)+d_{1}, \\
& \dot{x}_{\left(\beta_{1}+1\right)}=x_{\left(\beta_{1}+2\right)}, \\
& \ldots \\
& \dot{x}_{n}=f_{m}(x, u)+\tilde{f}_{m}(x, u)+d_{m},
\end{aligned}
$$

where $x, u$ are the system state vector, control input vector, respectively;

$x=\left[x_{1}, \ldots, x_{1}^{\left(\beta_{1}-1\right)}, \ldots, x_{\left(n-\beta_{m}+1\right)}, \ldots, x_{\left(n-\beta_{m}+1\right)}^{\left(\beta_{m}-1\right)}\right]^{T} \in R^{n}, \beta_{1}+\beta_{2}+\ldots+\beta_{m}=n, u=$ $\left[u_{1}, \ldots, u_{m}\right]^{T} \in R^{m}, f_{i}(i=1, \ldots, m)$ are known smooth nonlinear functions, $\tilde{f}_{i}(i=1, \ldots, m)$ are unknown uncertain nonlinearities of the system, and $d_{i}(\mathrm{i}=1,2, \ldots, \mathrm{m})$ denote the external disturbances.

Remark 1: There are many practical physical systems which can be described by the model (1), for example, the mass-spring-damper [18], the rotated inverted pendulum [19] and the n-link manipulator [20].

A reference model is as follows:

$$
\dot{x}_{r}(t)=A_{r} x_{r}(t)+r(t),
$$


where $x_{r}(t)$ is a reference state, $\mathrm{r}(\mathrm{t})$ is a bounded reference input, and $A_{r}$ is an asymptotically stable matrix.

Control objective: Design a controller to guarantee that the nonlinear system (1) is stable and the state can track the reference state $x_{r}(t)$.

The known part of the system (1) can be approximated by a fuzzy T-S model composed of $L$ rules. For convenience of research, fuzzy T-S model includes the external disturbance $d$. The ith rule of the fuzzy model is as follows:

$$
\begin{aligned}
& \text { IF } z_{1}(t) \text { is } F_{1}^{i} \text { and }, \ldots, \text { and } z_{s}(t) \text { is } F_{s}^{i}, \text { THEN } \\
& \dot{x}(t)=A_{i} x(t)+B_{i} u(t)+d, \quad i=1,2, \ldots, L,
\end{aligned}
$$

where $z_{1}(t), \ldots, z_{s}(t)$ are the premise variables, $F_{j}^{i}(\mathrm{j}=1,2, \ldots, \mathrm{s})$ are the fuzzy sets, $L$ is the number of IF-THEN rules, $A_{i}$ and $B_{i}$ are some constant matrices with compatible dimensions, $B_{i}=$ $\left[0, \ldots, b_{i 1}^{T}, \ldots, 0, \ldots, b_{i m}^{T}\right]^{T} \in R^{n \times m}$ with $b_{i 1} \in R^{m}, \ldots, b_{i m} \in R^{m}$, and $d=\left[0, \ldots, d_{1}, \ldots, 0, \ldots, d_{m}\right]^{T}$. The final output of the fuzzy system is inferred as follows:

$$
\dot{x}(t)=\sum_{i=1}^{L} \mu_{i} A_{i} x(t)+\sum_{i=1}^{L} \mu_{i} B_{i} u(t)+d,
$$

where

$$
\mu_{i}=\nu_{i}(z(t)) / \sum_{i=1}^{L} \nu_{i}(z(t)), \nu_{i}(z(t))=\prod_{j=1}^{S} F_{j}^{i}\left(z_{j}(t)\right),
$$

and $F_{j}^{i}\left(z_{j}(t)\right)$ is the grade of membership of $z_{j}(t)$ in $F_{j}^{i}$. Therefore, the approximating error for the nonlinear system (1) and the uncertainties of the nonlinear system (1) can be expressed as $B \Delta(x)$, where $B=\operatorname{diag}\left[B^{1}, \ldots, B^{m}\right], B^{i}=[0, \ldots, 0,1]^{T} \in R^{\beta_{i}}$ and $\Delta(x)=\left[\Delta_{1}, \ldots, \Delta_{m}\right]^{T}$.

Therefore, the nonlinear system (1) could be rearranged as

$$
\dot{x}(t)=\sum_{i=1}^{L} \mu_{i} A_{i} x(t)+\sum_{i=1}^{L} \mu_{i} B_{i} u(t)+B \Delta(x)+d .
$$

\section{Design of controller and stability analysis}

\subsection{Design of controller}

The controller is chosen as

$$
u(t)=u_{l}(t)-u_{f}(t),
$$

where $u_{l}(t)$ denotes the state-feedback controller based on fuzzy T-S model, $u_{f}(t)$ is the adaptive compensator based on fuzzy logic systems.

The state-feedback controller $u_{l}(t)$ based on fuzzy T-S model is designed as

$$
u_{l}(t)=\sum_{i=1}^{L} \mu_{i} K_{i}\left(x(t)-x_{r}(t)\right),
$$

where $u_{l}(t)$ is used to stabilize the linear part of the system $(1)$, and $K_{i}(\mathrm{i}=1,2, \ldots, \mathrm{L})$ are matrices with proper dimensions and satisfy

$$
\bar{A}_{i j}^{T}+P \bar{A}_{i j}+\frac{1}{\rho^{2}} P P+\bar{Q}<0, i, j=1,2, \ldots, L,
$$


where $\bar{A}_{i j}=\left[\begin{array}{cc}A_{i}+B_{i} K_{j} & -B_{i} K_{j} \\ 0 & A_{r}\end{array}\right], \bar{Q}=\operatorname{diag}\{2 Q, 2 Q\}, P$ and $Q$ are some symmetric and positive definite matrices, and $\rho$ is a positive constant.

The adaptive compensator based on fuzzy logic systems is given by

$$
u_{f}(t)=\left\{\begin{array}{l}
E^{-1} \hat{u}(x \mid \Theta), \quad \text { if } E \text { is nonsingular } \\
E^{T}\left(I+E E^{T}\right)^{-1} \hat{u}(x \mid \Theta) \text { if } E \text { is singular, }
\end{array}\right.
$$

which is used to compensate the approximating error and the uncertainties. $\operatorname{In}(10)$,

$$
E_{i}=\left[b_{i 1}^{T}, \ldots, b_{i m}^{T}\right]^{T} \in R^{m \times m}, E=\sum_{i=1}^{L} \mu_{i} E_{i},
$$

and $\hat{u}(x \mid \Theta)$ is constructed by fuzzy logic systems. The updating law of $\Theta$ is as follows:

$$
\dot{\Theta}=\eta_{1} \Psi^{T}(x) \bar{B}^{T} P x,
$$

where $\eta_{1}$ is a positive constant, $\Psi(x)$ is a fuzzy basis-function matrix, and the definition of $\Psi(x)$ is given in (16).

\subsection{Stability analysis}

Note that

$$
\begin{gathered}
\sum_{i=1}^{L} \mu_{i} B_{i} u_{f}(t)-B \Delta(x, x(t-\tau))=B\left(E u_{f}(t)-\Delta(x)\right) \\
=\left\{\begin{array}{l}
B(\hat{u}(x \mid \Theta)-\Delta(x)) \\
B\left(\hat{u}(x \mid \Theta)-\left(I+E E^{T}\right)^{-1} \hat{u}(x \mid \Theta)-\Delta(x)\right)
\end{array}\right. \\
\triangleq B(\hat{u}(x \mid \Theta)-\Delta(x)) .
\end{gathered}
$$

Substituting (7) into (6) yields

$$
\dot{x}(t)=\sum_{i=1}^{L} \mu_{i} A_{i} x(t)+\sum_{i=1}^{L} \sum_{j=1}^{L} \mu_{i} \mu_{j} B_{i} K_{j}\left(x(t)-x_{r}(t)\right)-B(\hat{u}(x \mid \Theta)-\Delta(x))+d .
$$

Denote $\tilde{x}(t)=\left[x^{T}(t), x_{r}^{T}(t)\right]^{T}$, and $\bar{B}=\left[\begin{array}{ll}B^{T} & 0\end{array}\right]^{T}$. From (2) and (14), a new extended closed-loop system is as follows:

$$
\dot{\tilde{x}}(t)=\sum_{i=1}^{L} \sum_{j=1}^{L} \mu_{i} \mu_{j} \bar{A}_{i j} \tilde{x}(t)+\bar{B}(-(\hat{u}(x \mid \Theta)-\Delta(x)))+d \prime,
$$

where $d \prime=\left[d^{T}, r^{T}(t)\right]^{T}$. When fuzzy logic systems $\hat{u}(x \mid \Theta)$ eliminate $\Delta(x)$, then the closedloop system (15) is stable. Thus, fuzzy logic systems are constructed to approximate the vector function $\Delta(x)$ as follows:

$$
\hat{\Delta}(x \mid \Theta)=\Psi(x) \Theta
$$

where 


$$
\Psi(x)=\operatorname{diag}\left[\xi_{1}^{T}(x), \ldots, \xi_{m}^{T}(x)\right], \Theta=\left[\theta_{1}^{T}, \theta_{2}^{T}, \ldots, \theta_{m}^{T}\right]^{T}
$$

in which $\theta_{i}(\mathrm{i}=1,2, \ldots, \mathrm{m})$ are the column vectors, and the weight $\Theta$ is an adaptive parameter. Define the optimal parameter estimation $\Theta^{*}$ as follows:

$$
\Theta^{*} \triangleq \arg \min _{\Theta \in \Omega}\left[\sup _{x \in U}\|\hat{\Delta}(x \mid \Theta)-\Delta(x)\|\right]
$$

where $U=\left\{x \in R^{n}\right\}, \Omega=\left\{\Theta \in R^{p m \times 1}\right\}$. U, $\Omega$ denote the sets of suitable bounds on $\mathrm{x}, \Theta$, respectively. Then the estimation error for the vector function $\Delta(x)$ can be expressed as

$$
\hat{\Delta}(x \mid \Theta)-\Delta(x)=\Psi(x) \tilde{\Theta}+w,
$$

where $w=\left[w_{1}, \ldots, w_{m}\right]^{T}$ is a residual term, $\tilde{\Theta}=\Theta-\Theta^{*}=\left[\left(\theta_{1}-\theta_{1}^{*}\right)^{T}, \ldots,\left(\theta_{m}-\theta_{m}^{*}\right)^{T}\right]^{T}$.

Denote $w^{\prime}=\left[\bar{w}^{T}, r^{T}(t)\right]^{t}$, and $\bar{w}=\left[0, \ldots, d_{1}-w_{1}, \ldots, 0, \ldots, d_{m}-w_{m}\right]^{T}$. Substituting (18) into (15), (15) is rearranged as

$$
\dot{\tilde{x}}(t)=\sum_{i=1}^{L} \sum_{j=1}^{L} \mu_{i} \mu_{j} \bar{A}_{i j} \tilde{x}(t)+\bar{B}(-\Psi(x) \tilde{\Theta})+w / .
$$

Theorem 1. For the nonlinear system (1), if the controller is chosen as (7) composed of the fuzzy state-feedback controller (8) and the adaptive compensator(10), and the updating law for the weight is chosen as (11), then the closed-loop system (15) is uniformly ultimately bounded (UUB) and the following tracking performance is achieved as

$$
\int_{0}^{T}\left(x(t)-x_{r}(t)\right)^{T} Q\left(x(t)-x_{r}(t)\right) d t \leq \tilde{x}^{T}(0) P \tilde{x}(0)+\frac{1}{\eta_{1}} \tilde{\Theta}^{T}(0) \tilde{\Theta}(0)+\rho^{2} \int_{0}^{T}\left(w^{\prime} w^{T}\right) d t
$$

where $\rho>0, \mathrm{P}, \mathrm{Q}$ are some symmetric and positive definite matrices.

Proof. Consider the following functional

$$
V=\frac{1}{2} \tilde{x}^{T} P \tilde{x}+\frac{1}{2 \eta_{1}} \tilde{\Theta}^{T} \tilde{\Theta}
$$

whose derivative can be computed as follows:

$$
\dot{V}=\frac{1}{2} \dot{\tilde{x}}^{T}(t) P \tilde{x}(t)+\frac{1}{2} \tilde{x}^{T}(t) P \dot{\tilde{x}}(t)+\frac{1}{\eta_{1}} \tilde{\Theta}^{T} \dot{\tilde{\Theta}}=\dot{V}_{1}+\dot{V}_{2},
$$

where

$$
\begin{gathered}
\dot{V}_{1}=\left(\sum_{i=1}^{L} \sum_{j=1}^{L} \mu_{i} \mu_{j} \bar{A}_{i j} \tilde{x}(t)\right)^{T} P \tilde{x}(t)+\tilde{x}^{T}(t) P\left(\sum_{i=1}^{L} \sum_{j=1}^{L} \mu_{i} \mu_{j} \bar{A}_{i j} \tilde{x}(t)\right)+\frac{1}{2} w^{T} P x(t)+\frac{1}{2} x^{T}(t) P w \prime \\
V_{2}=\left[\tilde { x } ^ { T } P \overline { B } \left(-(\Psi(x) \tilde{\Theta})+\frac{1}{\eta_{1}} \tilde{\Theta}^{T} \dot{\tilde{\Theta}}\right.\right. \\
\dot{V}_{1} \leq \frac{1}{2} \sum_{i=1}^{L} \sum_{j=1}^{L} \mu_{i} \mu_{j} \tilde{x}^{T}(t)\left(\bar{A}_{i j}^{T} P+P \bar{A}_{i j}+\frac{1}{\rho^{2}} P P\right) \tilde{x}(t)+\frac{1}{2} \rho^{2} w{ }^{T} w \prime
\end{gathered}
$$

Substituting (9) into (25) yields 


$$
\dot{V}_{1} \leq-\frac{1}{2} \tilde{x}^{T}(t) \bar{Q} \tilde{x}(t)+\frac{1}{2} \rho^{2} w^{T} w \prime
$$

From (11),(24)

$$
V_{2}=\left[\tilde{x}^{t} P \bar{B}\left(-(\Psi(x) \tilde{\Theta})+\frac{1}{\eta_{1}} \tilde{\Theta}^{T} \dot{\tilde{\Theta}}\right]=0\right.
$$

Thus

$$
\dot{V}=\dot{V}_{1}+\dot{V}_{2} \leq-\frac{1}{2} \tilde{x}^{T}(t) \bar{Q} \tilde{x}(t)+\frac{1}{2} \rho^{2} w^{T} w \prime
$$

When $\|e\|>\frac{\rho}{\lambda_{\min }(\bar{Q})}\|\bar{w}\|, \dot{V}<0$.Thus, the closed-loop system (15) is UUB.

Note that

$$
\begin{gathered}
\int_{0}^{T}\left(x(t)-x_{r}(t)\right)^{T} Q\left(x(t)-x_{r}(t)\right) d t=\int_{0}^{T}\left[x^{T}(t) x_{r}^{T}(t)\right]\left[\begin{array}{ll}
Q & -Q \\
-Q & Q
\end{array}\right]\left[x^{T}(t) x_{r}^{T}(t)\right]^{T} d t \\
\leq \int_{0}^{T}\left[x^{T}(t) x_{r}^{T}(t)\right] \operatorname{diag}\{2 Q, 2 Q\}\left[x^{T}(t) x_{r}^{T}(t)\right]^{T} d t=\int_{0}^{T} \tilde{x}^{T}(t) \bar{Q} \tilde{x}(t) d t
\end{gathered}
$$

Integrating the above inequality (28) from $\mathrm{t}=0$ to $\mathrm{T}$ yields $(20)$.

By Schur complements, the inequalities (9) are transformed into the linear matrix inequalities. Therefore, the common solution $P$ and $K_{j}(\mathrm{j}=1,2, \ldots, \mathrm{L})$ are required to be found. $P$ is chosen as the form $P=\operatorname{diag}\left\{P_{1}, P_{2}\right\}$, where $P_{1}, P_{2}$ are some symmetric and positive definite matrices. The inequalities (9) are equivalent to the following matrix inequalities

$$
\left[\begin{array}{lll}
S_{11} & -P_{1} B_{i} K_{j} & 0 \\
-\left(B_{i} K_{j}\right)^{T} P_{1} & S_{22} & P_{2} \\
0 & P_{2} & -\rho^{2} I
\end{array}\right]<0, i, j=1,2, \ldots, L
$$

where $S_{11}=P_{1}\left(A_{i}+B_{i} K_{j}\right)+\left(A_{i}+B_{i} K_{j}\right)^{T} P_{1}+\frac{1}{\rho^{2}} P_{1} P_{1}+2 Q$, and $S_{22}=P_{2} A_{r}+A_{r}^{T} P_{2}+2 Q$.

The matrix inequalities (30) imply $S_{11}<0$. Denote $\mathrm{W}=P_{1}^{-1}$ and $Y_{j}=K_{j} W . \quad S_{11}<0$ is equivalent to the linear matrix inequalities

$$
\left[\begin{array}{ll}
S & W \\
W & -(2 Q)^{-1}
\end{array}\right]<0, i, j=1,2, \ldots, L,
$$

where $S=A_{i} W+W A_{i}^{T}+B_{i} Y_{j}+\left(B_{i} Y_{j}\right)^{T}+\left(\rho^{2}\right)^{-1} I$.

$P_{1}$ and $K_{j}(j=1,2, \ldots, L)$ are obtained by (31). And then, substituting $P_{1}$ and $K_{j}(j=$ $1,2, \ldots, L)$ into $(30), P_{2}$ is obtained.

Remark 2: If a controlled system is fourth-order, the dimension of the matrix inequalities (30) is 12. By use of the method in [12], the dimension of the matrix inequalities (58) is 20. By use of the method in [9], the dimension of matrix inequalities in Theorem 1 is no less than 20. Thus, the dimension of matrix inequalities is reduced. 


\section{Simulation example}

Consider a 2-link manipulator system in [20]

$$
M(q) \ddot{q}(t)+C(q, \dot{q}) \dot{q}(t)+G(q)=\tau(t) .
$$

Consider the existence of the uncertainties and external disturbances in system (32). Thus, the plant is modified as follows:

$$
\ddot{q}(t)+C(q, \dot{q}) \dot{q}(t)+g(q)=B(q) u(t)+\sum_{i=1}^{r} \xi_{i}(t) q(t)+d \prime
$$

where $C(q, \dot{q})=H^{-1}(q) C \prime(q, \dot{q}), g(q)=H^{-1}(q) g \prime(q), B(q)=H^{-1}(q), d \prime=H^{-1}(q) d, q=$ $\left[q_{1}, q_{2}\right]^{T}$ and $u(t)=\left[u_{1}, u_{2}\right]^{T}=\tau(t) \cdot \xi_{i}(t)(i=1,2, \ldots, r)$ are uncertain and bounded. $d$ is random noise with zero mean and variance 0.05 , and $d$ is bounded.

The reference model is as follows:

$$
\dot{x}_{r}(t)=A_{r} x_{r}(t)+r(t),
$$

where

$$
A_{r}=\operatorname{diag}\left\{A_{r 1}, A_{r 2}\right\}, A_{r 1}=A_{r 2}=\left[\begin{array}{cc}
0 & 1 \\
-6 & -5
\end{array}\right], r(t)=\left[0, r_{1}(t), 0, r_{2}(t)\right]^{T} .
$$

Denote $x_{1}=q_{1}, x_{2}=\dot{q}_{1}, x_{3}=q_{2}, x_{4}=\dot{q}_{2}$. Fuzzy T-S model is used to approximate the nonlinear system at $x_{1}=-\frac{\pi}{2}, 0, \frac{\pi}{2}$ and $x_{3}=-\frac{\pi}{2}, 0, \frac{\pi}{2}$. The membership functions are adopted as triangle type. Fuzzy T-S model with Nine rules in the form (3) is given, where

$$
\begin{aligned}
& A_{1}=\left[\begin{array}{cccc}
0 & 1 & 0 & 0 \\
5.927 & -0.001 & -0.315 & -0.0000084 \\
0 & 0 & 0 & 1 \\
-6.859 & 0.002 & 3.155 & 0.0000062
\end{array}\right], A_{2}=\left[\begin{array}{cccc}
0 & 1 & 0 & 0 \\
3.0428 & -0.0011 & -0.1791 & -0.0002 \\
0 & 0 & 0 & 1 \\
-3.5436 & 0.0313 & 2.5611 & 0.0000114
\end{array}\right] \text {, } \\
& A_{3}=\left[\begin{array}{cccc}
0 & 1 & 0 & 0 \\
6.2728 & 0.003 & 0.4339 & -0.0001 \\
0 & 0 & 0 & 1 \\
-9.1041 & 0.0158 & -1.0574 & -0.000032
\end{array}\right], A_{4}=\left[\begin{array}{cccc}
0 & 1 & 0 & 0 \\
6.5434 & 0.0017 & 1.2427 & -0.0002 \\
0 & 0 & 0 & 1 \\
-3.1873 & 0.0306 & -5.1911 & -0.000018
\end{array}\right], \\
& A_{5}=\left[\begin{array}{cccc}
0 & 1 & 0 & 0 \\
11.1336 & 0 & -1.8145 & 0 \\
0 & 0 & 0 & 1 \\
-9.0918 & 0 & 9.1638 & 0
\end{array}\right], A_{6}=\left[\begin{array}{cccc}
0 & 1 & 0 & 0 \\
6.1702 & -0.001 & 1.687 & -0.0002 \\
0 & 0 & 0 & 1 \\
-2.3559 & 0.0314 & 4.5298 & -0.000011
\end{array}\right] \\
& A_{7}=\left[\begin{array}{cccc}
0 & 1 & 0 & 0 \\
6.1206 & 0.0041 & 0.6205 & 0.0001 \\
0 & 0 & 0 & 1 \\
8.8794 & 0.0193 & -1.0119 & 0.000044
\end{array}\right], A_{8}=\left[\begin{array}{cccc}
0 & 1 & 0 & 0 \\
3.6421 & -0.0018 & 0.0721 & 0.0002 \\
0 & 0 & 0 & 1 \\
2.429 & -0.0305 & 2.9832 & -0.000019
\end{array}\right]
\end{aligned}
$$




$$
\begin{gathered}
A_{9}=\left[\begin{array}{cccc}
0 & 1 & 0 & 0 \\
6.2933 & -0.0009 & 0.2188 & -0.000012 \\
0 & 0 & 0 & 1 \\
-7.4649 & 0.0024 & 3.2693 & -0.0000092
\end{array}\right], B_{1}=\left[\begin{array}{cccc}
0 & 1 & 0 & -1 \\
0 & -1 & 0 & 2
\end{array}\right]^{T}, B_{2}=\left[\begin{array}{cccc}
0 & 0.5 & 0 & 0 \\
0 & 0 & 0 & 1
\end{array}\right]^{T} \\
B_{3}=\left[\begin{array}{llll}
0 & 1 & 0 & 1 \\
0 & 1 & 0 & 2
\end{array}\right]^{T}, B_{4}=\left[\begin{array}{cccc}
0 & 0.5 & 0 & 0 \\
0 & 0 & 0 & 1
\end{array}\right]^{T} B_{5}=\left[\begin{array}{cccc}
0 & 1 & 0 & -1 \\
0 & -1 & 0 & 2
\end{array}\right]^{T}, B_{6}=\left[\begin{array}{cccc}
0 & 0.5 & 0 & 0 \\
0 & 0 & 0 & 1
\end{array}\right]^{T} \\
B_{7}=\left[\begin{array}{llll}
0 & 1 & 0 & 1 \\
0 & 1 & 0 & 2
\end{array}\right]^{T}, B_{8}=\left[\begin{array}{cccc}
0 & 0.5 & 0 & 0 \\
0 & 0 & 0 & 1
\end{array}\right]^{T} B_{9}=\left[\begin{array}{cccc}
0 & 1 & 0 & -1 \\
0 & -1 & 0 & 2
\end{array}\right]^{T} .
\end{gathered}
$$

Using the LMI box in Matlab, $K_{j}(j=1,2, \ldots, L)$ are obtained

$$
\begin{aligned}
& K_{1}=\left[\begin{array}{cccc}
-75.5707 & -41.2895 & -19.7728 & -8.9886 \\
4.5163 & -0.5944 & -49.7511 & -24.5727
\end{array}\right], K_{2}=\left[\begin{array}{cccc}
-76.4364 & -41.3132 & -13.7595 & -5.9976 \\
7.1834 & 1.0290 & -49.0587 & -24.2578
\end{array}\right] \text {, } \\
& K_{3}=\left[\begin{array}{cccc}
-75.4503 & -41.2461 & -19.8614 & -9.0371 \\
4.3907 & -0.6474 & -49.6965 & -24.54837
\end{array}\right], K_{4}=\left[\begin{array}{cccc}
-76.4364 & -41.3132 & -13.7595 & -5.9976 \\
7.1834 & 1.0290 & -49.0587 & -24.2578
\end{array}\right] \text {, } \\
& K_{5}=\left[\begin{array}{cccc}
-76.4364 & -41.3132 & -13.7595 & -5.9976 \\
7.1834 & 1.0290 & -49.0587 & -24.2578
\end{array}\right], K_{6}=\left[\begin{array}{cccc}
-76.4364 & -41.3132 & -13.7595 & -5.9976 \\
7.1834 & 1.0290 & -49.0587 & -24.2578
\end{array}\right], \\
& K_{7}=\left[\begin{array}{cccc}
-76.4364 & -41.9511 & -6.2666 & -2.2808 \\
11.1565 & 3.4005 & -48.7116 & -24.1209
\end{array}\right], K_{8}=\left[\begin{array}{cccc}
-76.4364 & -41.3132 & -13.7595 & -5.9976 \\
7.1834 & 1.0290 & -49.0587 & -24.2578
\end{array}\right] \text {, } \\
& K_{9}=\left[\begin{array}{cccc}
l c l-76.7763 & -42.0400 & -6.0715 & -2.1821 \\
-11.3875 & 3.4986 & -48.8204 & -24.1694
\end{array}\right],
\end{aligned}
$$

Then, the controller is given by

$$
u(t)=u_{l}(t)-u_{f}(t)
$$

where

$$
u_{l}(t)=\sum_{i=1}^{9} \mu_{i} K_{i}\left(x(t)-x_{r}(t)\right) \text { and } u_{f}=\left(\sum_{i=1}^{9} \mu_{i} E_{i}\right)^{-1} \hat{u}(x \mid \Theta)
$$

with the updating law (11). In (11), the symmetric and positive definite matrix

$$
P=\left[\begin{array}{cccc}
0.0070 & 0.0035 & -0.0004 & -0.0002 \\
0.0035 & 0.0021 & 0.0004 & 0.0001 \\
-0.0004 & 0.0004 & 0.0073 & 0.0032 \\
-0.0002 & 0.0001 & 0.0032 & 0.0017
\end{array}\right]
$$


Seven fuzzy rules are defined in adaptive fuzzy logic systems.

$R^{(j)}$ : if $x_{1}$ is $F_{1}^{j}, \ldots, x_{4}$ is $F_{4}^{j}$, then $y$ is $G^{j} \quad(\mathrm{j}=1,2, \ldots, 7)$,

$$
\begin{gathered}
\mu_{F_{i}^{1}}\left(x_{i}\right)=\frac{1}{1+\exp \left[5\left(x_{i}+0.8\right)\right]}(i=1,2, \ldots, 4), \quad \mu_{F_{i}^{1}}\left(x_{i}\right)=\exp \left[-\left(x_{i}+0.6\right)^{2}\right](i=1,2, \ldots, 4), \\
\mu_{F_{i}^{3}}\left(x_{i}\right)=\exp \left[-\left(x_{i}+0.4\right)^{2}\right](i=1,2, \ldots, 4), \quad \mu_{F_{i}^{4}}\left(x_{i}\right)=\exp \left[-\left(x_{i}\right)^{2}\right](i=1,2, \ldots, 4), \\
\mu_{F_{i}^{5}}\left(x_{i}\right)=\exp \left[-\left(x_{i}-0.4\right)^{2}\right](i=1,2, \ldots, 4), \quad \mu_{F_{i}^{6}}\left(x_{i}\right)=\exp \left[-\left(x_{i}-0.6\right)^{2}\right](i=1,2, \ldots, 4), \\
\mu_{F_{i}^{7}}\left(x_{i}\right)=\frac{1}{1+\exp \left[5\left(x_{i}-0.8\right)\right]}(i=1,2, \ldots, 4) . \\
\operatorname{Denote} S_{1}=\sum_{j=1}^{7} \sum_{i=1}^{4} \mu_{F_{i}^{j}}\left(x_{i}\right), \operatorname{then} \\
\xi(x)=\left[\prod_{i=1}^{4} \mu_{F_{i}^{1}}\left(x_{i}\right) / S_{1}, \ldots, \prod_{i=1}^{4} \mu_{F_{i}^{7}}\left(x_{i}\right) / S_{1}\right]=\left[\xi_{1}, \ldots, \xi_{7}\right], \Psi(x)=\operatorname{diag}\left[\xi^{T}(x), \xi^{T}(x)\right] .
\end{gathered}
$$

The initial condition is set to be

$$
\left(x_{1}(0), x_{2}(0), x_{3}(0), x_{4}(0), x_{r 1}(0), x_{r 2}(0), x_{r 3}(0), x_{r 4}(0)\right)=(0.4,0,-0.4,0,0,0,0,0) .
$$

Choose $r_{1}(t)=r_{2}(t)=4 \sin (t), r=2, \xi_{1}(t)=1+20 \sin (\mathrm{t}), \xi_{2}(\mathrm{t})=2(1-\exp (-\mathrm{t})) /(1+\exp (-\mathrm{t}))$ and the parameter $\eta_{1}=20$. Simulation results are shown in Fig.1- Fig.3.

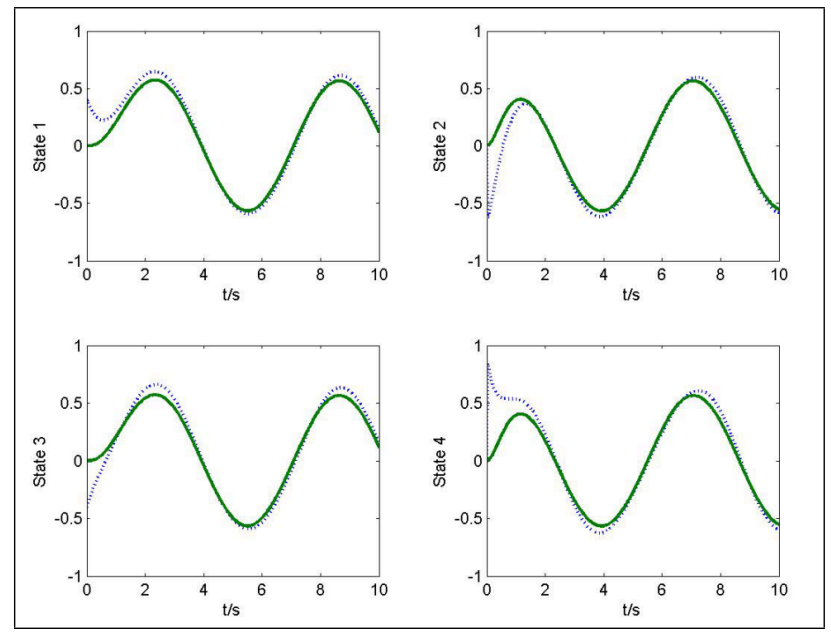

Figure 1: State responses of $x_{1}, x_{2}, x_{3}$ and $x_{4}$ (dotted line), $x_{r 1}, x_{r 2}, x_{r 3}$ and $x_{r 4}$ (solid line)

By only using the fuzzy controller based on T-S model [20], simulation result is shown in Fig. 4 and the tracking performance comparison between proposed method and approach in [20] is given in table 1. 


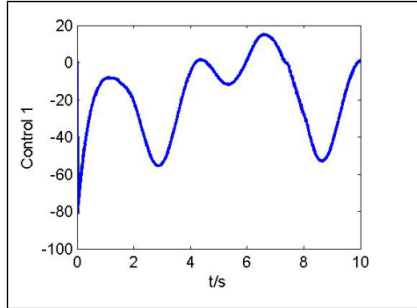

Figure 2: The control input $u_{1}$

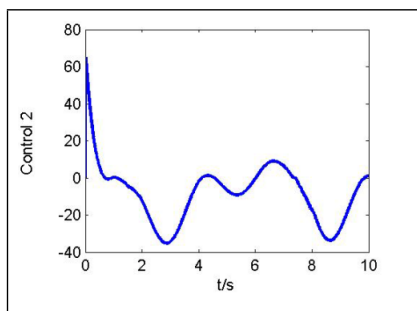

Figure 3: The control input $u_{2}$
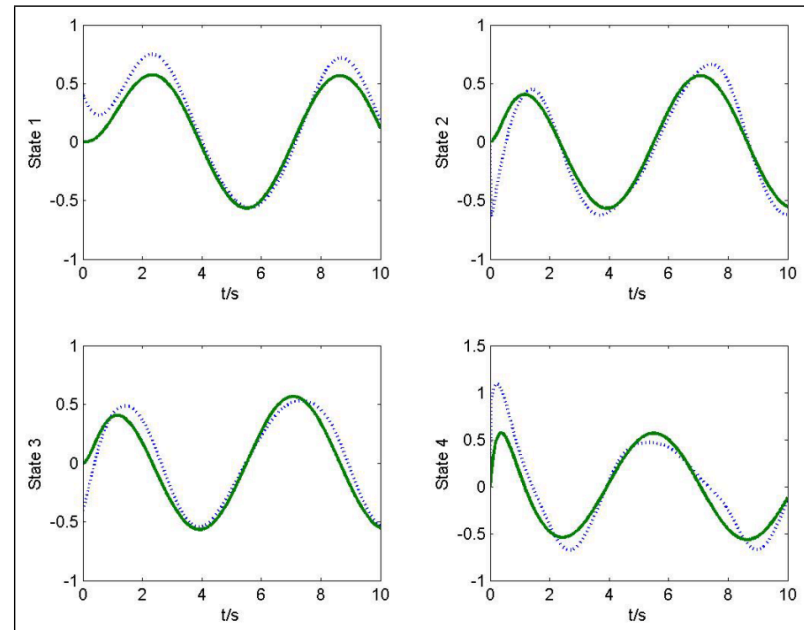

Figure 4: State responses of $x_{1}, x_{2}, x_{3}$ and $x_{4}$ (dotted line), $x_{r 1}, x_{r 2}, x_{r 3}$ and $x_{r 4}$ (solid line) 
Table 1: The tracking performance comparison between proposed method and approach in [20].

\begin{tabular}{|c|c|c|c|c|}
\hline$E_{i}=\int_{0}^{t}\left(x_{i}-x_{r i}\right)^{2} d t$ & $E_{1}$ & $E_{2}$ & $E_{3}$ & $E_{4}$ \\
\hline The proposed method & 0.0677 & 0.1338 & 0.0587 & 0.2177 \\
\hline The method in [20] & 0.1380 & 0.1760 & 0.1244 & 0.3343 \\
\hline
\end{tabular}

\section{Conclusion}

The developed controller makes full use of the advantages of two fuzzy models. Theory analysis verifies the feasibility of the proposed control scheme and simulation results demonstrate the effectiveness of the proposed control scheme.

\section{Acknowledgements}

This work is supported by the National Natural Science Foundation of China (60974028).

\section{Bibliography}

[1] K.Tanaka, M.Sugeno. Stability analysis and design of fuzzy control systems. Fuzzy Sets and Systems,1992,45(2):135-156.

[2] Bong-Jae Rhee, Sangchul Won. A new fuzzy Lyapunov function approach for a Takagi -Sugeno fuzzy control system design. Fuzzy Sets and Systems, 2006, 157(9): 1211 - 1228.

[3] H. K. Lam, Lakmal D. Seneviratne. Stability Analysis of Interval Type-2 Fuzzy-Model- Based Control Systems. IEEE Transactions on Systems, Man, and Cybernetics-Part B: Cybernetics,2008,38(3): 617-628.

[4] Sung Hyun Kim ,Poo Gyeon Park.Observer-Based Relaxed Ho Control for Fuzzy Systems Using a Multiple Lyapunov Function. IEEE Transactions on Fuzzy Systems, 2009,17(2):476484.

[5] Antonio Sala, Carlos Ariño.Relaxed Stability and Performance LMI Conditions for TakagiSugeno Fuzzy Systems With Polynomial Constraints on Membership Function Shapes. IEEE Transactions on Fuzzy Systems,2008,16(5):1328-1336.

[6] Miguel Bernal, Thierry Marie Guerra,Alexandre Kruszewski.A membership-function- dependent approach for stability analysis and controller synthesis of Takagi-Sugeno models. Fuzzy Sets and Systems,2009,160(19):2776-2795.

[7] Yan-Wu Wang,Zhi-Hong Guan,Hua O.Wang.Impulsive synchronization for Takagi-Sugeno fuzzy model and its application to continuous chaotic system.Physics Letters A, 2005,339(3$5): 325-332$.

[8] Shinn-Horng Chen, Wen-Hsien Ho, Jyh-Horng Chou.Robust Controllability of T-S FuzzyModel-Based Control Systems With Parametric Uncertainties. IEEE Transactions on Fuzzy Systems,2009,17(6):1324-1335.

[9] J.C.Lo, M.L.Lin. Robust nonlinear modeling and control via uncertain fuzzy systems. Fuzzy Sets and Systems,2004,143(2):189-209. 
[10] JunYoneyama.Robust $H \infty$ control analysis and synthesis for Takagi-Sugeno general uncertain fuzzy systems. Fuzzy Sets and Systems 2006,157(16):2205- 2223.

[11] Fuwen Yang,Yongmin Li,Set-Membership Fuzzy Filtering for Nonlinear Discrete- Time Systems. IEEE Transactions on Fuzzy Systems,2010,40(1):116-123.

[12] B.S.Chen, C.S.Tseng, H.J.Uang. Mixed fuzzy output feedback control design for nonlinear dynamic systems: an LMI approach. IEEE Transactions on Fuzzy Systems,2000,8(3): 249265.

[13] C.M.Park.LMI-based robust stability analysis for fuzzy feedback linearization regulators with its application.Information Sciences,2003,152:287-301.

[14] Changchun Hua, Qing-Guo Wang , Xinping Guan.Robust Adaptive Controller Design for Nonlinear Time-Delay Systems via T-S Fuzzy Approach. IEEE Transactions on Fuzzy Systems,2009,17(4):901-910.

[15] Guoliang Wei, Gang Feng, Zidong Wang . Robust H $\infty$ Control for Discrete-Time Fuzzy Systems With Infinite-Distributed Delays.IEEE Transactions on Fuzzy Systems,2009,17(1) 224-232.

[16] Li-Xin Wang. Stable adaptive fuzzy control of nonlinear systems. IEEE Transactions on Fuzzy Systems, 1993,1(3): 146-155.

[17] Weisheng Chen, Zhengqiang Zhang. Globally stable adaptive backstepping fuzzy control for output-feedback systems with unknown high-frequency gain sign. Fuzzy Sets and Systems, 2010, 161(6): 821-836.

[18] Huai-Ning Wu, Kai-Yuan Cai.H2 guaranteed cost fuzzy control for uncertain nonlinear systems via linear matrix inequalities. Fuzzy Sets and Systems, 2004,148(3):411-429.

[19] Y. Liu, S.S. Hu. Fuzzy Robust Tracking Control for Uncertain Nonlinear Systems. Acta Automatica Sinica. 2004,30(6):949-953.

[20] C. S. Tseng, B. S. Chen, H.J. Uang. Fuzzy tracking control design for nonlinear dynamic systems via T-S fuzzy model. IEEE Transactions on Fuzzy Systems,2001,9(3): 381-392. 